\title{
MIVIS image geocoding experience on merging position attitude system data and public domain GPS stream (ASI-GeoDAF)
}

\author{
Giovanni Avanzi $\left({ }^{1}\right)$, Angelo Palombo $\left({ }^{2}\right)$ and Stefano Pignatti $\left({ }^{2}\right)\left({ }^{3}\right)$ \\ (') VITROCISET SpA, Roma, Italy \\ $\left(^{2}\right)$ Laboratorio Aereo Ricerche Ambientali (LARA), IIA-CNR, Tor Vergata (RM), Italy \\ $\left(^{3}\right)$ Istituto di Metodologie per l'Analisi Ambientale (IMAA), CNR, Tito Scalo (PZ), Italy
}

\begin{abstract}
The use of airborne scanners involves geo-referencing problems, which are difficult because of the need to know the exact platform position and attitude for each scan line. The errors of the onboard navigation system are normally corrected using ground control point on the image. This post-processing correction procedure is too long in case of multiple flight campaigns, and besides it implies the need to have available 1:10000 orthophotoimages or maps in digital format. To optimize the above procedure a new method to correct MIVIS navigational data in the post-processing phase has been implemented. The procedure takes into consideration the GPS stream in Rinex format of common knowledge and findable on the web, acquired at the ground stations of the Geodetic Data Archiving Facilities provided by ASI. The application of this correction entails the assumption that the environmental variables affecting both onboard and geodetic GPS equally affect the position measurements. The airborne data correction was carried out merging the two data sets (onboard and ground station GPS) to achieve a more precise aircraft trajectory. The present study compares the geo-coded images obtained by means of the two post-processing methods.
\end{abstract}

Key words MIVIS - geocoding - geometric correction - GPS

\section{Introduction}

Remotely sensed imagery is often integrated with geo-coded data deriving from other sensors and cartographic information, therefore the integration process forces us to make images geometrically consistent with the chosen reference (Lechi, 1999).

Mailing address: Dr. Angelo Palombo, Istituto di Metodologie per l'Analisi Ambientale (IMAA), CNR, C.da S. Loja, 85050 Tito Scalo (PZ), Italy; e-mail: palombo@imaa.cnr.it
Hyperspectral sensors, based on the whiskbroom concept, are operated both from spaceborne and airborne platforms. The latter are more operationally flexible and often adjustable to special needs (Nieuwenhuis, 1993). Dealing with spaceborne imagery is easy because of a stable flight path, attitude and smaller scales of images. In this case geometric correction can be achieved by means of two-dimensional polynomial functions. However, in airborne applications, like the Multispectral Infrared and Visible Imaging Spectrometer (MIVIS), the sensor's movements are more complex due to irregular very frequent motions of the aircraft. To overcome the related problems airborne sensors are often mounted on steady platforms, which cannot fully compensate all disturbances. Additional distortions result from the topographic land re- 
lief. The geometric correction of image data can easily be achieved if the sensor's position and attitude can be measured with high precision using an Inertial Navigation System (INS) in combination with a Global Positioning System (GPS) receiver (Lithopoulos, 1999). However, since facts sometimes diverge from this ideal situation, many problems persist in practical applications. Although very precise mean sensor's position and attitude are available noise effects always occur, and consequently they have to be modeled for error estimation and quality management. Combined GPS/INS systems often are not available because of the high cost of those reaching a high precision.

In order to increase the MIVIS PAS accuracy, compass data and GPS data were postprocessed before being used in the geometric correction chain. In particular, as MIVIS PAS does not employ a differential GPD (DGPS) technology, data were pseudo-differentiated by means of a post-processing phase with the use of measurements recorded at a fixed station of a national network, supported by ASI within the GeoDAF project.

\section{MIVIS scanning geometry}

MIVIS is an airborne scanner that simultaneously records 102 different bands from the visible to the thermal infrared region. Due to its high spatial and spectral resolution, this sensor is a powerful instrument to discriminate the optical properties of the surfaces, and to study the bio-physical processes occurring on the Earth's surface.

The optical components of this instrument are the scan head, which is composed of a primary collecting telescope and a rotating scan mirror (placed at $45^{\circ}$ compared to the rotation axis). Each scan line is composed of 755 digitized pixels with a 12 bpp (bit per pixel) resolution. The white light arriving from the Earth, collimated by the scan head, is distributed by means of dichroic mirrors into 4 spectrometers recording the radiation coming from the Earth's surface at the same time.

The distinctive quantities of the MIVIS acquisition geometry are as follows:
- FoV $=71.06^{\circ}$ total Field of View;

- IFoV=2.0 mrad Instantaneous Field of View;

- sample rate (angular step) $=1.64 \mathrm{mrad}$;

- Scan Rotation Frequency (SRF): 25, 16.7, 12.5, 8.3, $6.25 \mathrm{~Hz}$.

From these values and based on geometric considerations, it derives that along a scan line the total ground cover is given by

$$
C=2 \cdot H \cdot \tan \left(\frac{\mathrm{FoV}}{2}\right)=1.43 \cdot H
$$

where $H$ represents the flight altitude. As the distance between the instrument and the ground varies along the scan line (fig. 1), it follows that the MIVIS spatial resolution decreases from the nadir to the extremities of the scan line (Avanzi et al., 1996). The pixel dimension along the scan line is determined by eq. (2.2) where $D x_{i}$ (dimension of pixel $i$ across scan line) and $D z_{i}$ (pixel dimension along the scan line) (Avanzi et al., 1995).

$$
D x_{i}=\frac{\mathrm{IFoV} \cdot H}{\cos ^{2}\left(\frac{|378-i| \cdot \mathrm{FoV}}{2}\right)}
$$

$$
D z_{i}=\frac{\mathrm{IFoV} \cdot H}{\cos \left(\frac{|378-i| \cdot \operatorname{FoV}}{2}\right)}
$$

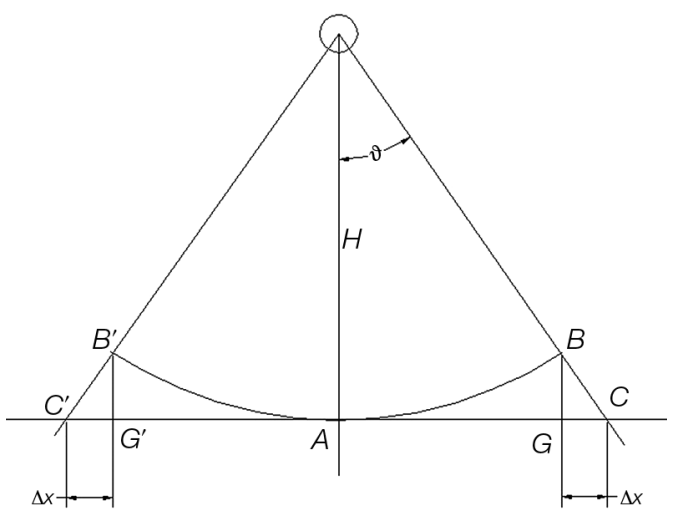

Fig. 1. Acquisition geometry, plan across the flight track. 
The pixel overlapping level in the two directions (parallel and orthogonal to the aircraft) depends on two distinct effects. In the direction orthogonal to the aircraft's motion it depends on the ratio IFoV and sample rate, which is an instrument's constant, while in the parallel direction it depends on the speed of the aircraft and its altitude (Daedalus, 1993).

The pixel distortion due to the dynamics of the platform is monitored by the MIVIS Position Attitude System (PAS). This system comprises a vertical gyroscope mounted on the scan head for measuring the roll and pitch angles of the platform; a Flux Gate Compass placed at the end of a wing of the aircraft for estimating the yaw angles compared to the magnetic north; and a GPS receiver within the digitizer for giving the aircraft's position and altitude in geographic coordinates as well as accurate time measurement. The precision of these instruments is respectively as follows:

GPS position accuracy $=\left\{\begin{array}{l} \pm 40 \mathrm{~m} \text { SA on } \\ \pm 15 \mathrm{~m} \text { SA off }\end{array}\right.$

Gyro accuracy $= \pm 0.2^{\circ}$

Compass accuracy $= \pm 0.6^{\circ}$.

There are some error sources inherent in GPS which degrade the system's accuracy resulting in slow drifts in the position solution for stationary receiver. These error sources are detailed in table I (Braasch, 2001). The Selective Availability (SA) is the greatest source of positioning errors for civilian users, causing errors of

Table I. The significant errors source in non-differential GPS applications as well now that selective availability has been deactivated.

\begin{tabular}{cc}
\hline \hline Error source & Per pseudorange rms (m) \\
\hline User-range error & $0-5$ \\
(sv clock and orbit) & \\
Ionospheric delay & $0-50$ \\
Tropospheric delay & $0-20$ \\
Noise & $0.2-3$ \\
Multipath & $0-10$ \\
Total & $0.2-55$ \\
\hline
\end{tabular}

up to $50 \mathrm{~m}$. The $\mathrm{SA}$ is a deliberate error introduced by the US Department of Defence for security reasons to degrade the position solution of non-military receivers (Cooper and DurrantWhite, 1994).

\section{Image distortion sources}

\subsection{Panoramic distortions}

The acquisition of the scan line implies its projection onto a cylindrical systems of radius equal to the platform's altitude (fig. 1). Since the ray-of-sight rotates across the flight direction around the sensor's projection center, each pixel is projected onto some part of a circle around the projection center. As a consequence, the pixel spatial resolution $\left(D x_{i}, D z_{i}\right)$ varies along the scan line, i.e. it decreases from the nadir (view angle equal to 0 ) towards the sides, as expressed in eq. (2.2) (Richards and Jia, 1999).

Figure 1 shows how at the side of the scan line the points at ground $C$ and $C^{1}$ are positioned on the image in $G$ and $G^{1}$. The two $G$ points correspond to the ground projection of the intersection of the circle of radius $\mathrm{H}$ with the straight lines pointing $C$ and $C^{1}$. On the scan line the real position $C$ and the apparent one $G$ are respectively determined as follows:

$$
\begin{aligned}
& \overline{A C}=H \cdot \tan \frac{\mathrm{FoV}}{2} \\
& \overline{A G}=H \cdot \sin \frac{\mathrm{FoV}}{2} .
\end{aligned}
$$

Taking into consideration the MIVIS FoV, from the above equation it follows that:

$$
\begin{aligned}
& \Delta x=\overline{A C}-\overline{A G}=H \cdot \tan \frac{\mathrm{FoV}}{2}+ \\
& -H \cdot \sin \frac{\mathrm{FOV}}{2}=0.14 \cdot H .
\end{aligned}
$$

\subsection{Platform-induced distortions}

The effects of the platform's movements (yaw, pitch, roll, drift, altitude and speed varia- 
tions) induce different types of distortions on the image plan. As to yaw variation, the single scan lines are not perpendicular to the flight track, with a consequent angular displacement of the object from its real position. As to pitch deviation, the scanner looks backward or forward instead of straight down, depending upon whether the pitch is nose down or nose up, with a consequent scan line overlapping or underlapping instead of being contiguous. As to roll variation, the scan line shifts to the right or to the left compared to the planned ground coverage, so resulting curved along the edge of the coverage; the roll-induced distortions are automatically corrected within a value of $\pm 10^{\circ}$. A horizontal plane drift may produce variations in flight direction, but without significant image distortions if it is long-lasting. Short-lasting drifts generally due to sudden wind gusts instead bring about shift distortions orthogonal to flight direction between scan lines. The altitude variations modify the image scale the increase or the decrease of the scan line swath. The errors associated with the GPS measurements affect the images like the pitch and roll distortions according to the error that could take place along or across the track direction.

\section{MIVIS geometric correction by means of Pas data}

Taking into consideration the reference system integrated with the aircraft, the following can be noticed: the $Z$ axis is oriented parallel to the aircraft's movement, the $Y$ axis is orthogonal to the aircraft's movement and parallel to the wings, while the $X$ axis is orthogonal to the other two axes and down-oriented, so forming a left-handed reference system as shown in fig. 2 . In this reference system the eq. (4.1) represents the $i$ pixel line of sight, which rotates of $70.06^{\circ}$ across the flight direction on the $X Y$ plane for covering the 755 positions of the scan line, therefore each single pixel is viewed with an angle of $1.64 \mathrm{mrad}$. The relation (4.1) represents the equation of the straight line of line of sight to the pixel and whose intersection with ground (i.e. $X=H$ ) locates the position of pixels $i$ along the scan line

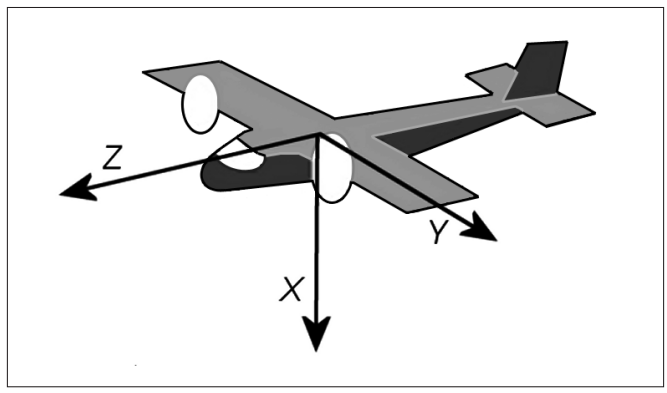

Fig. 2. Reference system integrated with the aircraft.

$$
Y_{i}=X \tan \left(\frac{\mathrm{FoV}}{2}-i \cdot \mathrm{IFoV}\right) .
$$

The value of $i$ equal to 0 points the first pixel acquired by the sensor corresponding to the outmost to the right with respect to the flight direction.

The pixel position, once determined with respect to the reference system integrated with the aircraft, has to be converted to a reference system integrated with the Earth. The coordinates deriving from the eq. (4.1) have been transformed into the geographical reference system by using the rotation matrixes $R$ reported as follows (Schläpfer et al., 1998):

$$
\begin{aligned}
R_{Z \vartheta} & =\left[\begin{array}{ccc}
\cos \vartheta & -\sin \vartheta & 0 \\
\sin \vartheta & \cos \vartheta & 0 \\
0 & 0 & 1
\end{array}\right] \text { roll; } \\
R_{Y \phi} & =\left[\begin{array}{ccc}
\cos \phi & 0 & \sin \phi \\
0 & 1 & 0 \\
-\sin \phi & 0 & \cos \phi
\end{array}\right] \text { pitch; } \\
R_{X \psi} & =\left[\begin{array}{ccc}
1 & 0 & 0 \\
0 & \cos \psi & -\sin \psi \\
0 & \sin \psi & \cos \psi
\end{array}\right] \text { yaw }
\end{aligned}
$$

the equation to be applied for the transformation of the coordinates is

$$
P_{i j}^{E}=P_{j}^{E}+R_{Z \vartheta} R_{Y \phi} R_{X \psi} P_{i j}^{A}
$$

where $P_{i j}^{E}$ is the geographical position ( $E$ apex) 


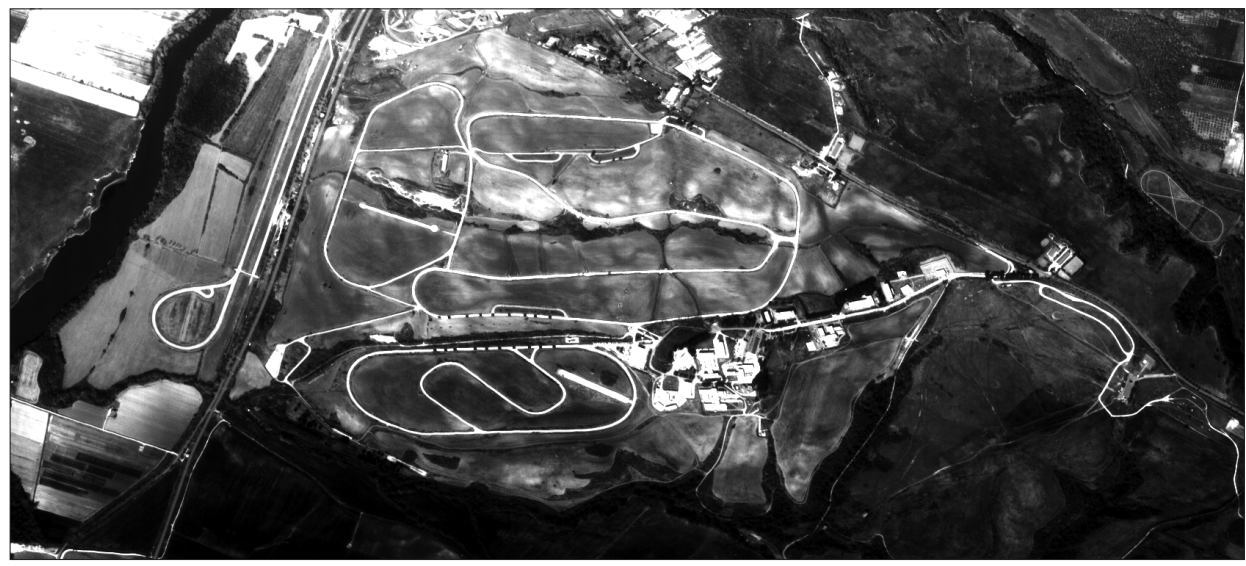

Fig. 3. Image before the correction.

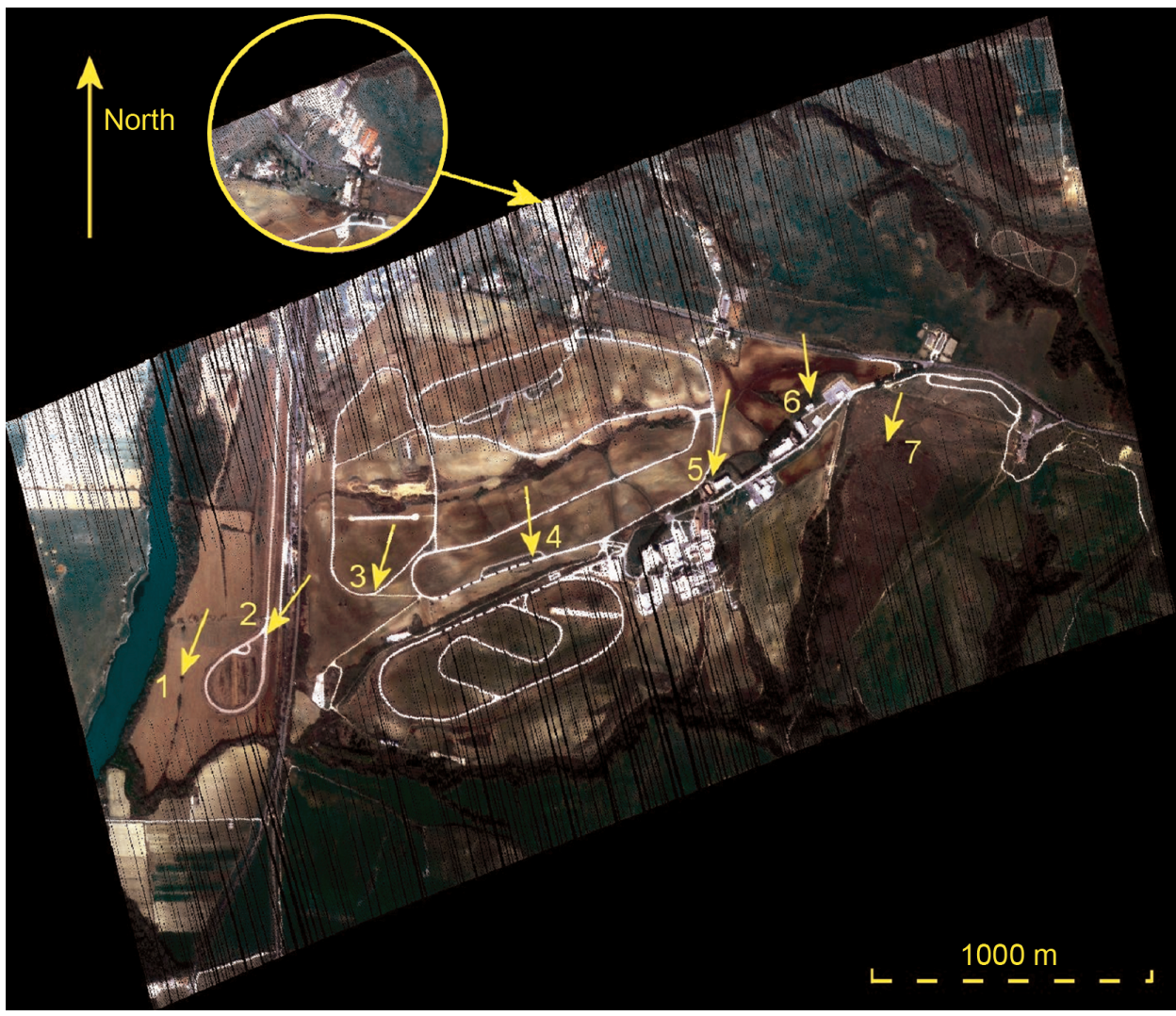

Fig. 4. GCP in the image after the geometric correction, the zoomed portion within the circle shows the image corrected with filtered compass. 
of $i$ pixel of line $j ; P_{j}^{E}$ is the geographical position of the aircraft for $j$ scan line measured by the PAS GPS; $P_{i j}^{A}$ is the position of the $i$ pixel along $j$ scan line in the aircraft's reference system ( $A$ apex) (Schläpfer et al., 2002). In the geo-coding of MIVIS data $\vartheta$ is equalized to 0 for roll values between $\pm 10^{\circ}$ in matrix $R_{Z \vartheta}$ because the digitizer automatically corrects the roll distortion. The pixel coordinates in the matrix $P_{i j}^{E}$ are produced as output in ASCII or binary format with extensions .GLT or .IGM to be used through ENVI software.

The PAS GPS resolves the position in latitude and longitude using the WGS84 system. Hence for geo-coding the images into a new cartographic reference system the GPS conversion into the requested system is just required. The images over Passo Corese used for this study in this paper have been in fact converted, using ENVI3.5, to metric units of the UTM N ZONE 33 system. An example of an image acquired on March 7, 2002 over Passo Corese (area close $40 \mathrm{~km}$ north to Rome) before and after the correction is shown in figs. 3 and 4.
Black lines disclosing a lack of pixels are visible on the corrected image along the side borders. Even though these could appear like a zone of under-sampled image they are related to the compass noise determining spurious frequencies in the yaw sequence as evidenced by Fourier Fast Transform (FFT) analysis. The FFT (fig. 5) of the compass highlights two frequencies at $4.1 \mathrm{~Hz}$ and $5.9 \mathrm{~Hz}$, that could not both be associated with the aircraft's swinging, so by filtering (band block) the two frequencies the effect on the under-sampled image borders vanished (zoomed area in fig. 4).

\section{GPS distortion correction}

Once the images have been corrected by means of navigational data, it should be possible to perfectly overlap two images acquired on the same area. Every shifting between the two images could be ascribed to errors connected to navigation instruments' measurements. The distance between the same Ground Control Points

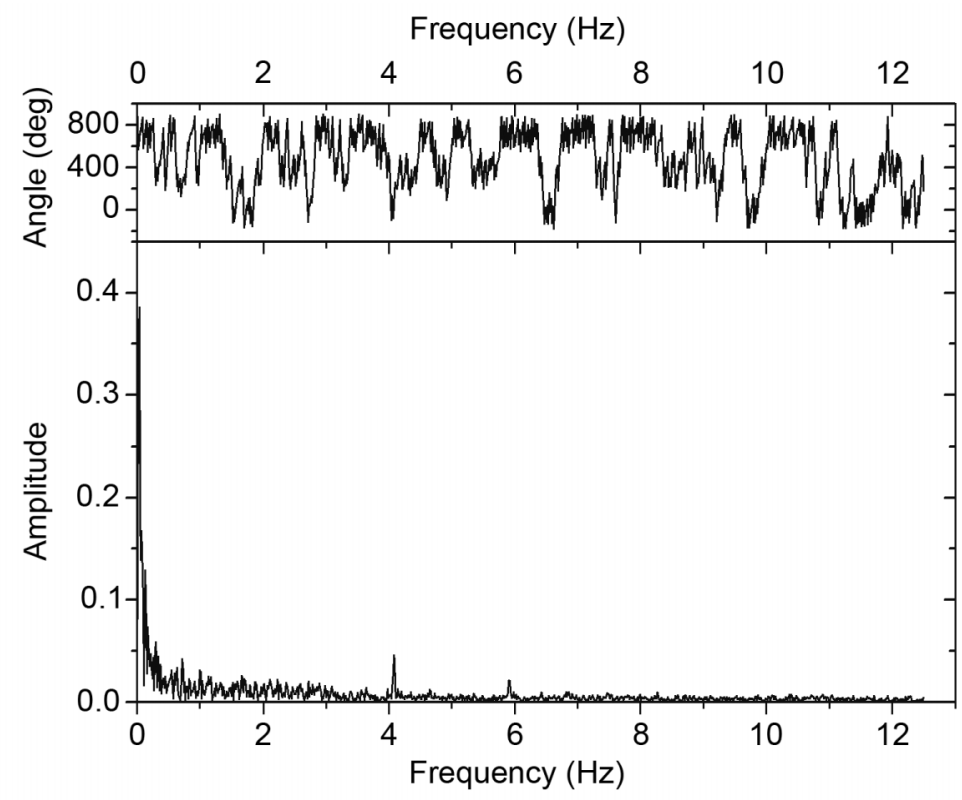

Fig. 5. The FFT of compass yaw with two peak at $4.1 \mathrm{~Hz}$ and $5.9 \mathrm{~Hz}$. 
(GCPs) selected in the two images was measured (fig. 4) to define the overlapping assessment of the two images, and then evaluate the navigation instruments' errors. To focus attention on the errors connected to GPS, the GCP in the nadir area of the two images have been selected since in these areas their errors are those mainly contributing to image distortion. These errors could be not related to INS sensors because: the contribution of the pitch is usually negligible in all the images; the contribution of the yaw is null at the center of the image and maximum at its borders; the aircraft's altitude variation does not involve deformations at the center of the image. The principal cause of uncertainty in the position of the MIVIS pixel at ground is due to the fact that MIVIS GPS is not DGPS. As a consequence, the geo-coding errors could be of about $50 \mathrm{~m}$. The possibility of differentiating MIVIS GPS in a post processing phase by using a fix reference station was tested in this paper. For this purpose GeoDAF (GeoData Archiving Facility) data of the GPS INGR station (owned by the Istituto Nazionale di Geofisica e Vulcanologia and located in Rome headquarters) were used for the MIVIS GPS post-processing.

The fixed station data were sampled during the MIVIS survey at $0.3 \mathrm{~Hz}$ and recorded in COMPACT RINEX FORMAT 1.0. Data were converted to WGS84 lat-long data and transformed into UTM N ZONE 33 by using PINNACLE commercial software. Figure 6 reports the $X-\bar{X}$ and $Y-\bar{Y}$ trends versus time in the period between the two flights, where $X$ and $Y$ are the UTM coordinates and $\bar{X}$ and $\bar{Y}$ are their daily averages. During the MIVIS survey the $X$ and $Y$ position drifts were $1.5 \mathrm{~m}$ and $3.5 \mathrm{~m}$ respectively.

To correct the GPS distortion which occurred during the MIVIS survey on Passo Corese, two images (run\#10 and run\#12), acquired respectively on March 7, 2002 at 11:47:23 UT and 12:00:27 UT were taken into consideration. Run\#10 was recorded at a flight altitude of $1560 \mathrm{~m}$ a.s.l. while run\#12 at $1050 \mathrm{~m}$. Among the runs the flight altitude difference is well visible in the image overlapping shown in fig. 7, as the scan lines of the run\#12 cover only a least land portion. For run\#10 (recording time of $\left.1^{\prime} 06^{\prime \prime}\right)$ three reference measures were taken by INGR, while for run\#12 (recording time of $\left.0^{\prime} 54^{\prime \prime}\right)$ two reference measures were made at the fixed station. Besides the concomitant measurements by INGR, previous and succeeding measurements were taken into consideration. Because of the different sampling rate, INGR variations were re-sampled to the MIVIS GPS time (seconds of the week). The MIVIS positions, corrected by subtracting the INGR variations, have been further used to geo-code

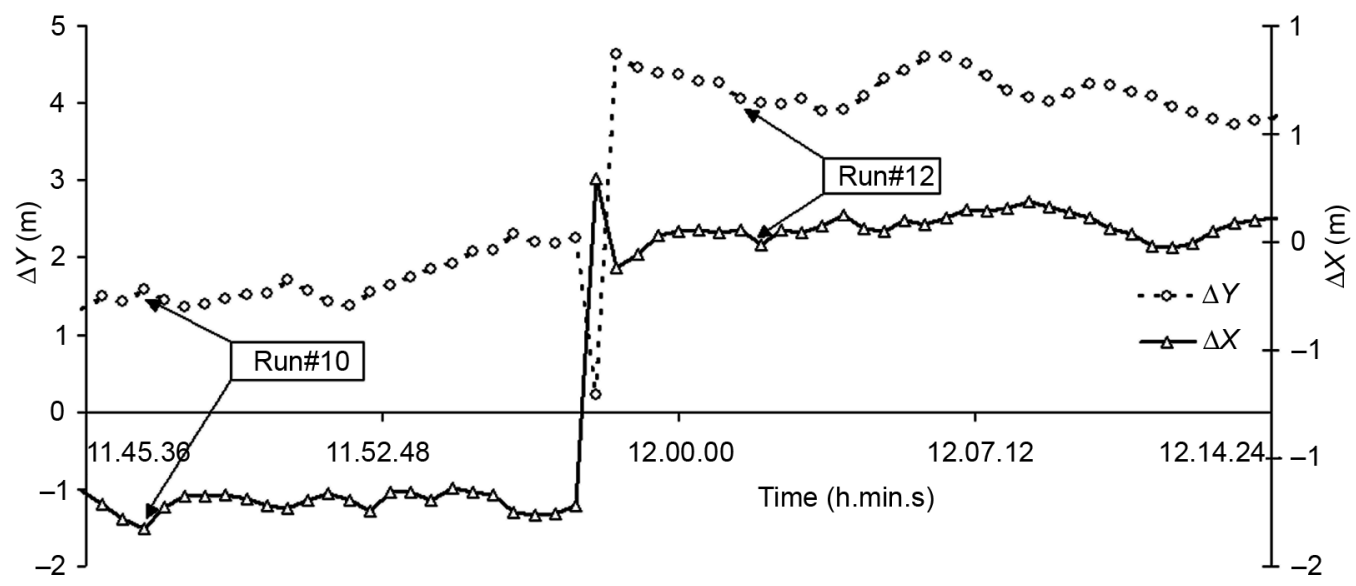

Fig. 6. UTM $X$ and $Y$ coordinate changes compared to their daily average value. 


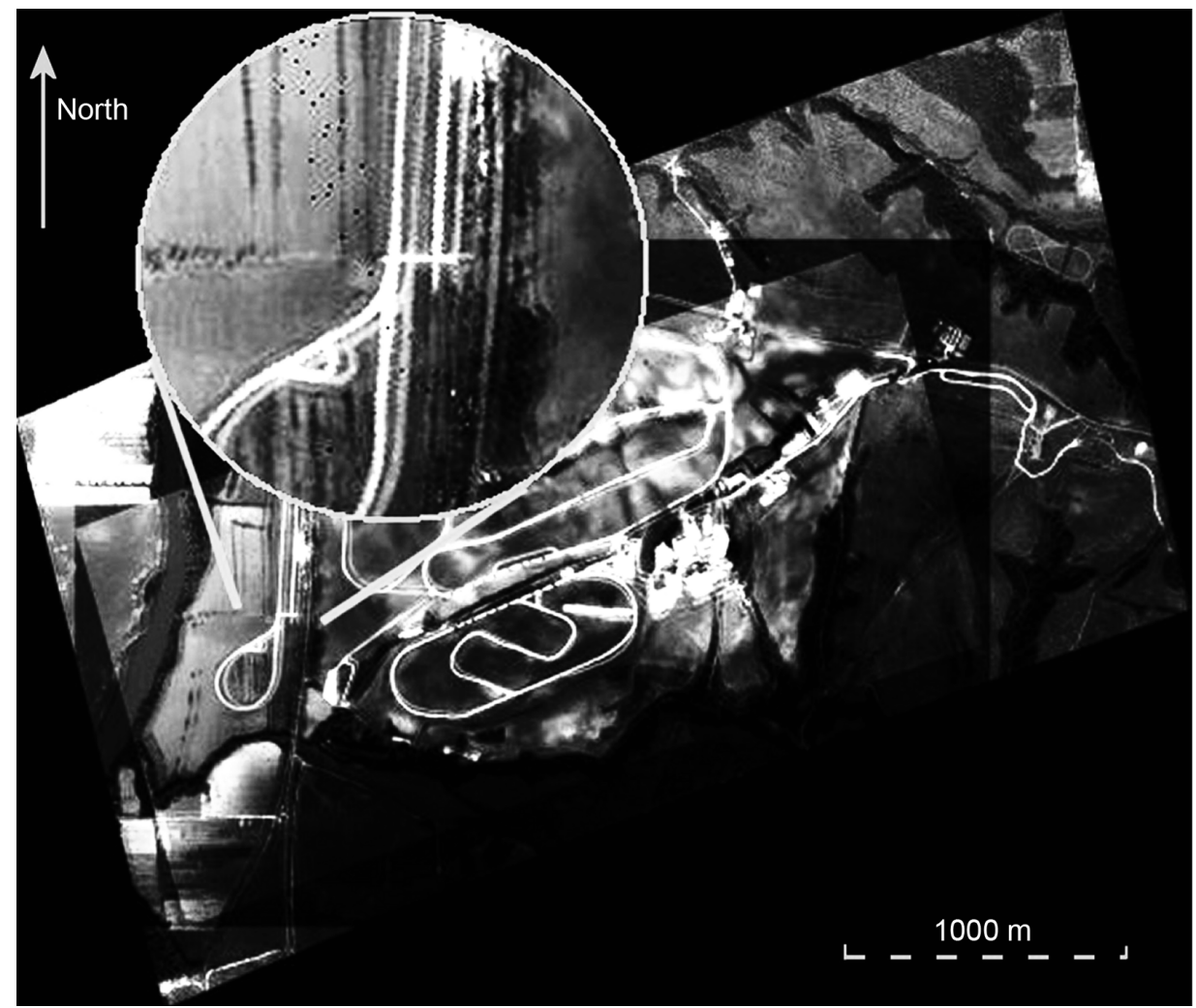

Fig. 7. Image 10 and 12 overlapped with the particular GCP.

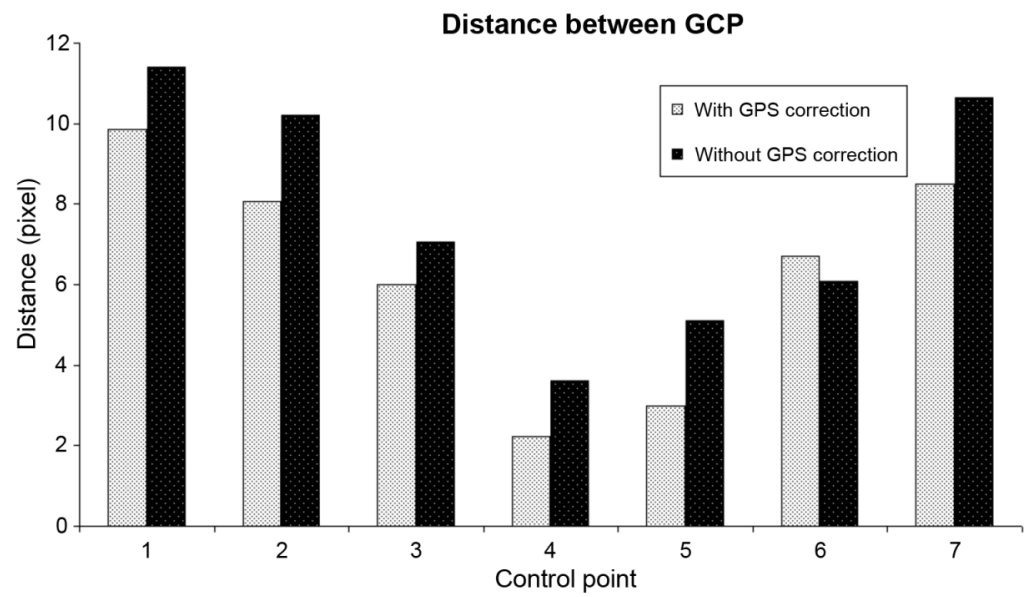

Fig. 8. The error of position calculated for the seven selected GCP. 
again run\#10 and \#12. Therefore by comparing the GCP shift on the two overlaps (run\#10/ run\#12) with and without PAS GPS correction, the increased accuracy in the geo-coded set, obtained with the post processed procedure, was then assessed. The improvement is shown in the graph of fig. 8, where the distances between GCP were measured before and after the correction. The mean distance calculated among all GCP decreases from 6.7 pixels to 5.5 pixels after the GPS correction (i.e. $20.1 \mathrm{~m}$ to $16.5 \mathrm{~m}$ ), then revealing an improvement of about $20 \%$. The obtained improvement is so low because the GPS SA was off at the time of the MIVIS survey on Passo Corese and therefore the GPS inaccuracy was determined just by the atmospheric interferences. Moreover, the accuracy level of the improvement is strictly related to the distance between the areal survey and the fixed GPS station as in the case of the application of DGPS technology (Harkleroad et al., 1990).

\section{Conclusions}

The GPS post processing correction method allows a trajectory refinement which reduces the pixel localization error within MIVIS images (evaluated in the nadir area). In the test area of Passo Corese the geo-coding accuracy improved by about $20 \%$ using the GPS measures of the INGR station within the GeoDAF Project. In any case, on the images sides the percentage correction decreases because other sources of errors are present (i.e. compass noise and topographic variations). In addition, using GeoDAF network the level of accuracy cannot be estimated in advance because it is strictly related to the distance between the station and the area surveyed. By way of GeoDAF data the error reduction is achieved without any data by additional instrumentation, because GeoDAF are attained using public domain data. Certainly this processing methodology could be more effective for MIVIS image geo-coding if ground data, recorded at $1 \mathrm{~Hz}$ corresponding to the MIVIS GPS acquisition frequency, are recorded.

Lastly, this procedure drastically decreases the numbers of the GCP to be acquired on car- tographic maps or orthophotos to obtain precise trajectory refinements and then avoid the application of the polynomial warping technique on images for a reliable spatial accuracy.

\section{Acknowledgements}

The authors are grateful to Maurizio Poscolieri for valuable discussions and to Angela Mirabelli for help in improving the paper.

\section{REFERENCES}

Avanzi, G., R. Bianchi, M. Capece, R.M. Cavalli, C.M. Marino and S. Pignatti (1995): Studio metodologico per la referenziazione geografica dei dati MIVIS, in Proceedings of the 'VII Convegno Nazionale AIT', 367-372

Avanzi, G., R. Bianchi, R.M. Cavalli, L. Fiumi, C.M. MARINO and S. PIGNATTI (1996): Use of MIVIS navigation data for precise aircraft positioning and attidute estimation, SPIE Proc., 2960, 184-192.

BRAASCH, M.S. (2001): Performance comparison of multipath mitigating receiver architectures, in Proceedings of the IEEE Aerospace Conference, vol. 3, 1309-1315.

COOPER, S. and H. DuRRANT-WhYTE (1994): A Kalman filter model for GPS navigation of land vehicles, in World' IROS '94 Proceedings, vol. 1, 157-163

Daedalus (1993): Manuale Daedalus. Operator Manual AA5000 Multispectral Infrared and Visibile Imaging Spectrometer (MIVIS), vol. 1.

HARKLEROAD, G., W. TANG and N. JOHNSON (1990): Estimation of errors correlation distance for differential GPS operation, in Position Location and Navigation Symposium, Record 'The 1990's - A Decade of Excellence in the Navigation Sciences', IEEE Plans '90, 378-382.

LeChi, G.M. (1999): Dispense del Corso di Telerilevamento (Facoltà di Ingegneria, Università degli Studi di Milano)

Lithopoulos, E. (1999): The applanix approach to GPS/INS integration, in Photogrammetric Week 99, edited by D. FRITSCH and R. SPILLER (Wichmann Verlag, Heidelberg), 53-57

NieuwenhuIs, G.J.A. (1993): Agrohydrological application of remote sensing, in Land Observation by Remote Sensing - Theory and Applications, edited by H.G. Buiten and J.G.P.W. Clevers (Gordon and Breach Science Publishers), 373-384.

RichARDS, J.A. and X. JiA (1999): Remote Sensing Digital Image Analysis - An Introduction (Springer Verlag, New York), 3rd edition, 42-52.

SCHLÄPFER, D. and R. RICHTER (2002): Geo-atmospheric processing of airborne imaging spectrometry data, Part 1. Parametric orthorectification, Int. J. Remote Sensing, 23 (13), 2609-2630.

SCHLÄPFER, D., M.E. SCHAEPMAN and K.I. ITTEN (1998): PARGE: PARametric GEocoding based on GCP-calibrated auxiliary data, in SPIE Int. Symp. on Opt. Sc, Eng. and Instr., San Diego (CA), 334-344. 